\title{
LATTICE STRUCTURES AND SPREADING MODELS
}

\author{
S. J. DILWORTH, E. ODELL AND B. SARI
}

\begin{abstract}
We consider problems concerning the partial order structure of the set of spreading models of Banach spaces. We construct examples of spaces showing that the possible structure of these sets include certain classes of finite semi-lattices and countable lattices, and all finite lattices.
\end{abstract}

\section{Contents}

\begin{tabular}{|l|r|}
\hline 0. & Introduction \\
\hline 1. & Background, questions and observations \\
\hline 2. Spreading model sets without a minimum element & 1 \\
\hline 3. Countable lattices with a minimum element & 2 \\
\hline References & 7 \\
\hline
\end{tabular}

\section{INTRODUCTION}

The spreading models of a Banach space $X$ usually have a simpler and better structure, both individually and collectively, than the class of subspaces of $X$. Sometimes knowledge of the spreading models can be used to deduce subspace knowledge about $X$ itself (e.g., [AOST, OS1] ) but the relationship is still not completely understood. Spaces with no "nice" subspaces can have very nice spreading models (e.g., [AD]).

In this paper we explore further the relationship between a space and its spreading models. In particular we study the possible partial order structures of the spreading models of $X$ generated by normalized weakly null sequences. In $\S 1$ we recall what is known and unknown and present some new structural observations along with the relevant background. In $\S 2$ and $\S 3$ we construct spaces $X$ with certain prescribed spreading model structures. In $\S 2$ we construct for each $n \in \mathbb{N}$, a space $X_{n}$ with $\left(S P_{w}\left(X_{n}\right), \leq\right)$ order isomorphic to $(\mathcal{P}(n) \backslash\{\emptyset\}, \subseteq)$ where $\mathcal{P}(n)$ is the power set of $\{1, \ldots, n\}$. In $\S 3$ we show that if $L$ is a countable lattice

Research of the second named author was partially supported by the National Science Foundation. The third named author had a visiting appointment at the University of South Carolina for the 2004-05 academic year during part of his research. 
with a minimum element not containing an infinite strictly increasing sequence, then there exists a reflexive space $X_{L}$ with $\left(S P_{w}\left(X_{L}\right), \leq\right)$ order-isomorphic to $L$. The construction uses some beautiful classical work of Lindenstrauss and Tzafriri [LT] on Orlicz sequence spaces.

\section{BACKGROUND, QUESTIONS AND OBSERVATIONS}

We use standard Banach space notation and terminology as in [LT].

Let $X$ be a separable infinite-dimensional Banach space. A normalized basic sequence $\left(x_{i}\right) \subseteq X$ generates a spreading model $\left(\tilde{x}_{i}\right)$ if for some $\varepsilon_{n} \downarrow 0$ for all $n \in \mathbb{N}$ and $\left(a_{i}\right)_{1}^{n} \subseteq[-1,1]$,

$$
\left(1+\varepsilon_{n}\right)^{-1}\left\|\sum_{i=1}^{n} a_{i} \tilde{x}_{i}\right\| \leq\left\|\sum_{i=1}^{n} a_{i} x_{k_{i}}\right\| \leq\left(1+\varepsilon_{n}\right)\left\|\sum_{i=1}^{n} a_{i} \tilde{x}_{i}\right\|
$$

for all $n \leq k_{1}<\cdots<k_{n}$. $\left(\tilde{x}_{i}\right)$ is a basic sequence which is 1-spreading and suppression-1 unconditional if $\left(x_{i}\right)$ is weakly null. Every normalized basic sequence has a subsequence which generates a spreading model (see $[\mathrm{BL}$ ) for these and more elementary facts about spreading models).

We let $\left[\left(\tilde{x}_{i}\right)\right]$ denote the equivalence class of all spreading models of $X$ which are equivalent (see below) to $\left(\tilde{x}_{i}\right) . S P_{w}(X)$ denotes the set of all such $\left[\left(\tilde{x}_{i}\right)\right]$ where we restrict ourselves only to spreading models generated by weakly null sequences. If $S P_{w}(X)=\emptyset$ then $X$ is a Schur space, so every normalized spreading model of $X$ is equivalent to the unit vector basis of $\ell_{1}$ by Rosenthal's $\ell_{1}$ theorem $[\mathrm{R}]$.

If $\left[\left(\tilde{x}_{i}\right)\right],\left[\left(\tilde{y}_{i}\right)\right] \in S P_{w}(X)$ we write $\left[\left(\tilde{x}_{i}\right)\right] \leq\left[\left(\tilde{y}_{i}\right)\right]$ if for some $C<\infty,\left(\tilde{y}_{i}\right) C$-dominates $\left(\tilde{x}_{i}\right)$, i.e., for all $\left(a_{i}\right) \subseteq \mathbb{R}$

$$
\left\|\sum a_{i} \tilde{x}_{i}\right\| \leq C\left\|\sum a_{i} \tilde{y}_{i}\right\|
$$

$\left(\tilde{x}_{i}\right)$ and $\left(\tilde{y}_{i}\right)$ are equivalent if each dominates the other. $\left(S P_{w}(X), \leq\right)$ is a partially ordered set.

We sometimes have occasion to consider a specific $\left(\tilde{x}_{i}\right)$ and shall abuse notation by writing "let $\left(\tilde{x}_{i}\right) \in S P_{w}(X)$." $c_{00}$ denotes the linear space of finitely supported real sequences.

Fact 1.1. AOST $\left(S P_{w}(X), \leq\right)$ is a semi-lattice, i.e., each two elements of $S P_{w}(X)$ admit a least upper bound. Moreover if $\left(\tilde{x}_{i}\right),\left(\tilde{y}_{i}\right) \in S P_{w}(X)$ there exists $\left(\tilde{z}_{i}\right) \in S P_{w}(X)$ which is 2-equivalent to the subsymmetric norm on $c_{00}$ given by

$$
\left\|\left(a_{i}\right)\right\|=\left\|\sum a_{i} \tilde{x}_{i}\right\| \vee\left\|\sum a_{i} \tilde{y}_{i}\right\|
$$

Fact 1.2. AOST Every countable subset of $\left(S P_{w}(X), \leq\right)$ admits an upper bound. Moreover if $\left(\tilde{x}_{i}^{n}\right)_{i=1}^{\infty} \in S P_{w}(X)$ for $n \in \mathbb{N}$ and $\left(C_{n}\right)_{n=1}^{\infty} \subseteq(0,1)$ with $\sum_{n=1}^{\infty} C_{n}^{-1} \leq 1$ then there 
exists $\left(\tilde{z}_{i}\right) \in S P_{w}(X)$ which $C_{n}$-dominates $\left(\tilde{x}_{i}^{n}\right)$ for each $n \in \mathbb{N}$. In addition for $\left(a_{i}\right) \in c_{00}$

$$
\left\|\sum a_{i} \tilde{z}_{i}\right\| \leq C_{1}^{-1}\left(\sum_{n=1}^{\infty} C_{n}^{-1}\left\|\sum a_{i} \tilde{x}_{i}^{n}\right\|\right) .
$$

We shall designate this $\left(\tilde{z}_{i}\right)$ by the notation $\left(\tilde{z}_{i}\right)=\left(\sum C_{n}^{-1} \tilde{x}_{i}^{n}\right)$, which in fact is motivated by the proof in AOST] (the precise quantification as given above is noted in [S2]).

Fact 1.3. S2] If $S P_{w}(X)$ admits an infinite strictly increasing sequence then $S P_{w}(X)$ is uncountable. In fact there exist $\left[\left(\tilde{y}_{i}^{\alpha}\right)\right] \in S P_{w}(X)$ for $\alpha<\omega_{1}$ so that $\left[\left(\tilde{y}_{i}^{\alpha}\right)\right]<\left[\left(\tilde{y}_{i}^{\beta}\right)\right]$ if $\alpha<\beta<\omega_{1}$.

Our next result is motivated by the proof of Fact 1.3

Theorem 1.4. Let $I$ be an infinite set and let $\left(\tilde{x}_{i}^{\alpha}\right)_{i=1}^{\infty} \in S P_{w}(X)$ for $\alpha \in I$. For $A \subseteq I$ define a subsymmetric norm on $c_{00}$ by $R_{A}\left(a_{i}\right)=\sup _{\alpha \in A}\left\|\sum a_{i} \tilde{x}_{i}^{\alpha}\right\|$. If for every non-empty finite $F \subseteq I, R_{I}$ is not equivalent to $R_{F}$, then $S P_{w}(X)$ admits an infinite strictly increasing sequence.

Proof. We may assume $I=\mathbb{N}$. We shall construct a strictly increasing sequence $\left(\tilde{y}_{i}^{n}\right)_{i=1}^{\infty}$ for $n \in \mathbb{N}$. We shall let

$$
\left(\tilde{y}_{i}^{1}\right)=\left(\sum_{n=1}^{\infty} 2^{-n} \tilde{z}_{i}^{n}\right) \quad \text { (see Fact 1.2) }
$$

where $\left(\tilde{z}^{n}\right)_{n=1}^{\infty}$ is a reordering of $\left(\tilde{x}^{n}\right)_{n=1}^{\infty}$ selected as follows. Let $\varepsilon_{n} \downarrow 0$ and for each $n \in \mathbb{N}$, $\tilde{z}^{2 n}$ is chosen so that for some $\left(a_{\ell}^{n}\right)_{\ell=1}^{\infty} \in c_{00}$,

$$
R_{I}\left(a_{\ell}^{n}\right)_{\ell=1}^{\infty}=1, \quad\left\|\sum_{\ell} a_{\ell}^{n} \tilde{z}_{\ell}^{2 n}\right\|>\frac{1}{2}
$$

and $R_{I_{n}}\left(a_{\ell}^{n}\right)_{\ell=1}^{\infty}<\varepsilon_{n} 2^{-2 n}$ where

$$
I_{n}=\left\{m \in \mathbb{N}: \tilde{x}^{m}=\tilde{z}^{j} \text { for some } j \leq 2 n-1\right\} .
$$

$\tilde{z}^{n}$ for $n$ odd is selected arbitrarily so as to exhaust the collection $\left(\tilde{x}^{s}\right)_{s \in \mathbb{N}}$.

For $n \in \mathbb{N}$ we have (see Fact 1.2 )

$$
\begin{aligned}
\left\|\sum_{\ell} a_{\ell}^{n} \tilde{y}_{\ell}^{1}\right\| & \leq 2 R_{I_{n}}\left(a_{\ell}^{n}\right)_{\ell=1}^{\infty}+2 \cdot 2^{-2 n}\left\|\sum_{\ell} a_{\ell}^{n} \tilde{z}_{\ell}^{2 n}\right\|+2 \sum_{m>2 n} 2^{-m}\left\|\sum_{\ell} a_{\ell}^{n} \tilde{z}_{\ell}^{m}\right\| \\
& <2 \varepsilon_{n} 2^{-2 n}+2 \cdot 2^{-2 n}+2 \sum_{m>2 n} 2^{-m} \\
& =\left(2 \varepsilon_{n}+4\right) 2^{-2 n} .
\end{aligned}
$$

Furthermore

$$
\left\|\sum a_{\ell}^{n} \tilde{y}_{\ell}^{1}\right\| \geq 2^{-2 n}\left\|\sum_{\ell} a_{\ell}^{n} \tilde{z}_{\ell}^{2 n}\right\|>\frac{1}{2} 2^{-2 n} .
$$


We thus obtain that $\left(\tilde{x}_{i}^{n}\right)<\left(\tilde{y}_{i}^{1}\right)$ for all $n \in \mathbb{N}$ and $\left(\tilde{y}_{i}^{1}\right)<R_{I}$. Moreover we can iterate the argument beginning anew with the collection $\left\{\left(\tilde{y}_{i}^{1}\right)\right\} \cup\left\{\left(\tilde{x}_{i}^{n}\right)\right\}_{n=1}^{\infty}$, which satisfies the same hypothesis as $\left.\left(\tilde{x}_{i}^{n}\right)\right\}_{n=1}^{\infty}$, to obtain $\tilde{y}^{2}$, and so on.

Fact 1.5. $S P_{w}(X)$ can be hereditarily uncountable AOST, i.e., $S P_{w}(Y)$ is uncountable for all infinite-dimensional subspaces $Y$ of $X$. If $S P_{w}(X)$ is countable, then by a diagonal argument one can find $X_{0} \subseteq X$ with $S P_{w}\left(X_{0}\right)=S P_{w}(Y)$ for all $Y \subseteq X_{0}$. It may be that then $\left|S P_{w}\left(X_{0}\right)\right|=1$ but this remains open.

We also have the

Problem 1.6. If $X$ is reflexive and $S P_{w}(X)$ is countable must some $\left(\tilde{x}_{i}\right) \in S P_{w}(X)$ be equivalent to the unit vector basis of $c_{0}$ or $\ell_{p}$ for some $1 \leq p<\infty$ ?

If so this would be a case where one would have a stronger theorem than Krivine's $[\mathrm{K}]$. Not every reflexive space has a spreading model isomorphic to $c_{0}$ or some $\ell_{p}$ ([OS2], [AOST] ). In the nonreflexive case it is possible to have $\left|S P_{w}(X)\right|=1$ yet the unique spreading model is not $c_{0}$ or any $\ell_{p}$. This is the case for certain Lorentz sequence spaces $d_{w, 1}$ (see $\S 2$ ).

Problem [1.6] was raised and partially solved in the case $\left|S P_{w}(X)\right|=1$ in [AOST]. We give some further partial results below.

Remark 1.7. Assume that $S P_{w}(X)$ is countable or more generally does not admit an infinite strictly increasing sequence. For $\left[\left(\tilde{x}_{i}\right)\right] \in S P_{w}(X)$ and $\left(a_{i}\right) \in c_{00}$, define

$$
R\left(a_{i}\right) \equiv R_{\left[\left(\tilde{x}_{i}\right)\right]}\left(a_{i}\right)=\sup \left\{\left\|\sum_{1}^{n} a_{i} \tilde{y}_{i}\right\|:\left(\tilde{y}_{i}\right) \in\left[\left(\tilde{x}_{i}\right)\right]\right\} .
$$

By $1.4 R$ is equivalent to $\left(\tilde{x}_{i}\right)$. Thus for each $\left(\tilde{y}_{i}\right) \in\left[\left(\tilde{x}_{i}\right)\right]$ there exists $C<\infty$ so that $\left(\tilde{y}_{i}\right)$ $C$-dominates every $\left(\tilde{z}_{i}\right) \in\left[\left(\tilde{x}_{i}\right)\right]$. Also $\left[\mathbf{S 2}\right.$ there exists $p=p\left(\tilde{x}_{i}\right) \in[1, \infty]$ so that for all $1 \leq q<p$ there exists $C_{q}<\infty$ so that for all $\left(a_{i}\right) \subseteq \mathbb{R}$,

$$
\left(\sum\left|a_{i}\right|^{p}\right)^{1 / p} \leq R\left(a_{i}\right) \leq C_{q}\left(\sum\left|a_{i}\right|^{q}\right)^{1 / q} .
$$

$p\left(\tilde{x}_{i}\right)$ is the infimum of the "Krivine $p$ 's" for $\left(\tilde{x}_{i}\right)$ (see [S2]). It is mistakenly stated in [S2] that, in this case, $p\left(\tilde{x}_{i}\right)$ is the only Krivine $p$. However, this is not yet clear.

Remark 1.8. Let $S P_{w}(X)$ be stabilized hereditarily for $X$. Then for all $\left(\tilde{x}_{i}\right) \in S P_{w}(X)$ there exist $X_{0} \subseteq X$ and $C<\infty$ such that: for all $Y \subseteq X_{0}$ there exists $\left(\tilde{y}_{i}\right) \in S P_{w}(Y)$ which is $C$-equivalent to $\left(\tilde{x}_{i}\right)$.

The proof is elementary. Assume not and use a diagonal argument to get a contradiction. 
Theorem 1.9. Suppose that $S P_{w}(X)$ is countable and that $S P_{w}\left(Y^{*}\right)$ is countable for all infinite-dimensional subspaces $Y$ of $X$. Then every $\left(\tilde{e}_{i}\right) \in S P_{w}(X)$ is equivalent to the unit vector basis of $c_{0}$ or $\ell_{p}$ for some $1 \leq p<\infty$.

Proof. Let $\left(e_{i}\right)$ be a normalized weakly null sequence in $X$ generating the spreading model $\left(\tilde{e}_{i}\right)$. By passing to a subsequence and renorming we may assume that $\left(e_{i}\right)$ is bimonotone basic and Schreier-unconditional, i.e. for some $\varepsilon_{n} \downarrow 0$ and all $F \in S_{1}$ and $\left(a_{i}\right) \in c_{00}$,

$$
\left\|\sum_{i \in F} a_{i} e_{i}\right\| \leq\left(1+\varepsilon_{\min F}\right)\left\|\sum a_{i} e_{i}\right\|
$$

(see [01]). Here $F \in S_{1}$ (first Schreier class) if $|F| \leq \min F$.

We may assume that no subsequence of $\left(e_{i}\right)$ is equivalent to the unit vector basis of $c_{0}$. Thus by passing to a further subsequence we may assume that $\left(f_{i}\right)$, the sequence of biorthogonal functions to $\left(e_{i}\right)$, is weakly null in $\left[\left(e_{i}\right)\right]^{*}$ O2 , Cor. 4.4]. From (1.1) it is easy see that $\left(f_{i}\right)$ is normalized and has spreading model $\left(\tilde{f}_{i}\right)$ which is 1-equivalent to $\left(\tilde{e}_{i}^{*}\right)$, the biorthogonal functionals to $\left(\tilde{e}_{i}\right)$ in $\left[\left(\tilde{e}_{i}^{*}\right)\right]$. By Krivine's theorem $[\mathrm{K}]$ it suffices to prove that, for some $D<\infty$, every spreading model $\left(\tilde{x}_{i}\right)$ of an identically distributed block basis $\left(x_{i}\right)$ of $\left(e_{i}\right)$ with $\left\|x_{i}\right\| \rightarrow 1$ is $D$-equivalent to $\left(\tilde{e}_{i}\right)$. Note that $\left(x_{i}\right)$ is weakly null and $\left(\tilde{x}_{i}\right)$ is equivalent to an identically distributed normalized block basis of $\left(\tilde{e}_{i}\right)$ and hence to $\left(\tilde{e}_{i}\right)$. Since $S P_{w}(X)$ is countable, by Theorem 1.4 there exists $C_{1}<\infty$ (which depends only on $\left.\tilde{e}_{i}\right)$ ) such that

$$
\left\|\sum a_{i} \tilde{x}_{i}\right\| \leq C_{1}\left\|\sum a_{i} \tilde{e}_{i}\right\| \quad\left(\left(a_{i}\right) \in c_{00}\right) .
$$

We may choose an identically distributed block basis $\left(g_{i}\right)$ of $\left(f_{i}\right)$ with $\operatorname{supp}\left(g_{i}\right) \subseteq \operatorname{supp}\left(x_{i}\right)$, $\left\|g_{i}\right\| \rightarrow 1$, and $g_{i}\left(x_{i}\right) \rightarrow 1$. Note that $\left(g_{i}\right)$ has spreading model $\left(\tilde{g}_{i}\right)$ which is 1-equivalent to an identically distributed block basis of $\left(\tilde{f}_{i}\right)$. Also $\left(g_{i}\right)$ is weakly null and since $S P_{w}\left(X^{*}\right)$ is countable we have, again by Theorem [1.4 that there exists $C_{2}<\infty$ (which depends only on $\left.\left(\tilde{f}_{i}\right)\right)$ such that

$$
\left\|\sum a_{i} \tilde{g}_{i}\right\| \leq C_{2}\left\|\sum a_{i} \tilde{f}_{i}\right\| \quad\left(\left(a_{i}\right) \in c_{00}\right) .
$$

Let $h_{i}$ be the restriction of $g_{i}$ to $\left[\left(x_{i}\right)\right]$. Since $\left(x_{i}\right)$ is bimonotone and Schreier unconditional, we have as above that $\left(h_{i}\right)$ has spreading model $\left(\tilde{h}_{i}\right)$ in $\left[\left(x_{i}\right)\right]^{*}$ which is 1-equivalent to $\left(\tilde{x}_{i}^{*}\right)$, the biorthogonal functionals to $\left(\tilde{x}_{i}\right)$. Thus for $\left(a_{i}\right) \in c_{00}$,

$$
\left\|\sum a_{i} \tilde{x}_{i}^{*}\right\|=\left\|\sum a_{i} \tilde{h}_{i}\right\| \leq\left\|\sum a_{i} \tilde{g}_{i}\right\| \leq C_{2}\left\|\sum a_{i} \tilde{f}_{i}\right\|
$$

By duality,

$$
\left\|\sum a_{i} \tilde{x}_{i}\right\| \geq \frac{1}{C_{2}}\left\|\sum a_{i} \tilde{e}_{i}\right\| \quad\left(\left(a_{i}\right) \in c_{00}\right)
$$


Thus by (1.2) and (1.3),$\left(\tilde{x}_{i}\right)$ is $D \equiv C_{1} C_{2}$-equivalent to $\left(\tilde{e}_{i}\right)$.

Theorem 1.10. Let $X$ be reflexive with $\left|S P_{w}(X)\right|=\left|S P_{w}\left(X^{*}\right)\right|=1$. Assume also that the element of $S P_{w}\left(X^{*}\right)$ is equivalent to the biorthogonal functionals of the element $\left(\tilde{x}_{i}\right)$ in $S P_{w}(X)$. Then $\left(\tilde{x}_{i}\right)$ is equivalent to the unit vector basis of $c_{0}$ or $\ell_{p}$ for some $1 \leq p<\infty$.

Proof. We first note that if $X_{0}$ is any infinite-dimensional subspace of $X$ then $X_{0}$ satisfies the same hypothesis as $X$. Indeed the only question here is the uniqueness of the spreading models in $X_{0}^{*}$. Let $\left(\tilde{f}_{i}\right)$ be a normalized spreading model for $X_{0}^{*}$ generated by $\left(f_{i}\right)$. Then $\left(f_{i}\right)$ is the image under the quotient map of a seminormalized weakly null sequence in $X^{*}$ and this yields that $\left(\tilde{x}_{i}^{*}\right)$ dominates $\left(\tilde{f}_{i}\right)$. A similar argument applied to the sequence biorthogonal to $\left(f_{i}\right)$ shows that $\left(\tilde{f}_{i}\right)$ dominates $\left(\tilde{x}_{i}^{*}\right)$. The result now follows from Theorem 1.9

The proof of Theorem 1.9 contains the following result.

Theorem 1.11. Let $\left(e_{i}\right)$ be a normalized basis for a reflexive space $X$ which is $C$-Schreier unconditional for some $C<\infty$, i.e.,

$$
\left\|\sum_{F} a_{i} e_{i}\right\| \leq C\left\|\sum a_{i} e_{i}\right\| \text { for all } F \in S_{1}
$$

and $\left(a_{i}\right) \subseteq \mathbb{R}$. If $\left|S P_{w}(X)\right|=\left|S P_{w}\left(X^{*}\right)\right|=1$ then the unique spreading model of $X$ is equivalent to the unit vector basis of $c_{0}$ or $\ell_{p}$ for some $1 \leq p<\infty$.

Remark 1.12. If $S P_{w}(X)$ is countably infinite then $S P_{w}(X)$ contains $\left\{\left(\tilde{x}_{i}^{n}\right)_{i=1}^{\infty}: n \in \mathbb{N}\right\}$ with either $\left(\tilde{x}_{i}^{n}\right)>\left(\tilde{x}_{i}^{m}\right)$ for all $n<m$ or $\left(\tilde{x}_{i}^{n}\right)$ and $\left(\tilde{x}_{i}^{m}\right)$ mutually incomparable for all $n \neq m$. Indeed Ramsey's theorem yields a subsequence of any sequence of spreading models satisfying either one of the two possibilities above or a sequence that is strictly increasing. The latter is ruled out by Fact 1.3. Both possibilities can occur for reflexive spaces. As noted elsewhere AOST (see also Theorem 3.7 below) it is easy to check that every spreading model of $\left(\sum \oplus \ell_{p_{n}}\right)_{p_{1}}$ is equivalent to some $\ell_{p_{n}}$ if $p_{1}<p_{2}<\cdots$. In $\S 3$ we shall show the second (mutually incomparable) possibility.

The uncountable case is less clear.

Problem 1.13. If $S P_{w}(X)$ is uncountable must there exist $\left\{\left(\tilde{x}_{i}^{\alpha}\right)_{i=1}^{\infty}: \alpha<\omega_{1}\right\} \subseteq S P_{w}(X)$ which is either strictly increasing w.r.t. $\alpha$, strictly decreasing or consists of mutually incomparable elements.

If there is a counterexample, $X$, say, to this question, then by Fact $1.3 S P_{w}(X)$ cannot contain an infinite increasing sequence. We do not know however the answer to this generalized version of Problem 1.13 
Problem 1.14. Let $L$ be an uncountable semi-lattice which admits no infinite strictly increasing sequence. Must $L$ admit a family $\left(x_{\alpha}\right)_{\alpha<\omega_{1}}$ with either

(i) $\forall \alpha \neq \beta, x_{\alpha}$ and $x_{\beta}$ are incomparable or

(ii) $\forall \alpha<\beta<\omega_{1}, x_{\alpha}>x_{\beta}$ ?

The following example due to Sierpinski (see [ER]) provides a counterexample to the corresponding question for posets. Let $L=\left(\left\{x_{\alpha}\right\}_{\alpha<\omega_{1}}, \preceq\right)$, where $\left\{x_{\alpha}\right\}_{\alpha<\omega_{1}}$ are distinct points in $(0,1)$ with $x_{\alpha} \prec x_{\beta}$ iff $\alpha>\beta$ and $x_{\alpha}>x_{\beta}$ (in $\mathbb{R}$ ). Then $L$ is a poset without any infinite increasing sequences and without any uncountable chains or antichains.

If an $\omega_{1}$-Suslin tree exists then Problem 1.14 easily has a negative answer. In a related result Shelah $\underline{\mathrm{Sh}}$ has shown that under $(\mathrm{CH})$ there exists an uncountable Boolean algebra without uncountable chains or antichains and moreover, $(\mathrm{CH})+$ no $\omega_{1}$-Suslin tree is consistent with ZFC. In particular, under $(\mathrm{CH})$ there is a counterexample to Problem 1.13 if $S P_{w}(X)$ is replaced by a general semi-lattice.

Our work in the next two sections suggests the following.

Problem 1.15. Let $L$ be a countable semi-lattice not admitting an infinite strictly increasing sequence. Does there exist $X$ (possibly even reflexive) with $\left(S P_{w}(X), \leq\right)$ orderisomorphic to $L$ ?

\section{Spreading model Sets Without a minimum Element}

In this section we shall construct some families of Banach spaces whose spreading model sets do not have a minimum element in the domination ordering. The Banach spaces in question are finite direct sums of certain Lorentz sequence spaces $d(w, p)$.

The construction depends on the existence of an arbitrary number of incomparable submultiplicative functions. We begin with a technical definition to facilitate the discussion.

Definition 2.1. Let $2 \leq n_{0} \leq \infty$ and let $S$ be a real-valued function defined on $\left[1, n_{0}\right]$. We shall say that $S$ is submultiplicative on $\left[1, n_{0}\right]$ (or on $[1, \infty)$ if $n_{0}=\infty$ ) if $S$ satisfies the following conditions:

(a) $S$ is piecewise-linear, continuous, strictly increasing, and concave.

(b) $S(x)=x$ for $1 \leq x \leq 2$.

(c) $S(x y) \leq S(x) S(y)$ for all $x, y$ such that $1 \leq x, y, x y \leq n_{0}$.

Lemma 2.2. Suppose that $2 \leq n_{0}<\infty$ and that $S$ is submultiplicative on $\left[1, n_{0}\right]$. Then there exists $\varepsilon_{0}>0$ such that for all $0<\varepsilon<\varepsilon_{0}$ the extension $S_{\varepsilon}$ of $S$ to the interval $\left[1, n_{0}^{2}\right]$ 
defined by

$$
S_{\varepsilon}(x)= \begin{cases}S(x) & \text { for } 1 \leq x \leq n_{0} \\ S\left(n_{0}\right)+\varepsilon\left(x-n_{0}\right) & \text { for } n_{0}<x \leq n_{0}^{2}\end{cases}
$$

is submultiplicative on $\left[1, n_{0}^{2}\right]$.

Proof. Since $S$ is continuous, piecewise-linear, and strictly increasing on $\left[1, n_{0}\right]$, there exists $c>0$ such that

$$
S(x) \geq S(x-h)+c h \quad\left(1 \leq x-h \leq x \leq n_{0}\right) .
$$

Define $\tilde{S}$ on $\left[1, n_{0}^{2}\right]$ as follows:

$$
\tilde{S}(x):=\inf \left\{S(a) S(b): x=a b, 1 \leq a, b \leq n_{0}\right\} .
$$

Since $S$ is continuous and strictly increasing on $\left[1, n_{0}\right]$ it follows that $\tilde{S}$ is continuous and strictly increasing on $\left[1, n_{0}^{2}\right]$. Moreover, conditions (b) and (c) of Definition 2.1] imply that $\tilde{S}(x)=S(x)$ for all $x \in\left[1, n_{0}\right]$. Suppose that $x:=n_{0}+h$ satisfies $n_{0} \leq x \leq n_{0}^{2}$. By compactness there exist $a_{x}, b_{x}$ such that $\tilde{S}(x)=S\left(a_{x}\right) S\left(b_{x}\right), x=a_{x} b_{x}$, and $1 \leq a_{x} \leq b_{x} \leq$ $n_{0}$. Then

$$
S\left(n_{0}\right)=\tilde{S}\left(n_{0}\right) \leq S\left(a_{x}\right) S\left(b_{x}-\frac{h}{a_{x}}\right)
$$

(since $n_{0}=a_{x}\left(b_{x}-h / a_{x}\right)$ and $S$ is submultiplicative on $\left[1, n_{0}\right]$ )

$$
\leq S\left(a_{x}\right)\left(S\left(b_{x}\right)-\frac{c h}{a_{x}}\right)
$$

(by (2.1) )

$$
\begin{aligned}
& =\tilde{S}\left(n_{0}+h\right)-\frac{c S\left(a_{x}\right)}{a_{x}} h \\
& \leq \tilde{S}\left(n_{0}+h\right)-\frac{c}{n_{0}} h,
\end{aligned}
$$

(since $S\left(a_{x}\right) \geq 1$ and $\left.a_{x} \leq n_{0}\right)$. So

$$
S\left(n_{0}\right)+\frac{c}{n_{0}} h \leq \tilde{S}\left(n_{0}+h\right) .
$$

Hence, provided $\varepsilon<c / n_{0}$, we have $S_{\varepsilon}(x) \leq \tilde{S}(x)$ for $1 \leq x \leq n_{0}^{2}$. To verify submultiplicativity of $S_{\varepsilon}$ on the interval $\left[1, n_{0}^{2}\right]$ it remains to check that

$$
S_{\varepsilon}(x y) \leq S_{\varepsilon}(x) S_{\varepsilon}(y)
$$

for all $1 \leq x \leq n_{o}$ and $n_{0} \leq y \leq n_{0}^{2}$ such that $x y \leq n_{0}^{2}$. Since $S_{\varepsilon}(x y)=S_{\varepsilon}(y)+\varepsilon(x y-y)$, we require

$$
\frac{S_{\varepsilon}(y)+\varepsilon(x-1) y}{S_{\varepsilon}(y)} \leq S_{\varepsilon}(x),
$$


i.e.

$$
\varepsilon(x-1) y \leq\left(S_{\varepsilon}(x)-1\right) S_{\varepsilon}(y) .
$$

First consider the case $x \geq 2$. Then $S_{\varepsilon}(x)-1 \geq 1$ and since $(x-1) y \leq x y \leq n_{0}^{2}$ it follows that (2.2) will be satisfied provided $n_{0}^{2} \varepsilon \leq S\left(n_{0}\right)$. On the other hand, if $1 \leq x \leq 2$, then by condition (b) of Definition 2.1] (2.2) reduces to $\varepsilon y \leq S_{\varepsilon}(y)$, which will again be satisfied provided $n_{0}^{2} \varepsilon \leq S\left(n_{0}\right)$. This proves the lemma for

$$
\varepsilon_{0}=\min \left(\frac{c}{n_{0}}, \frac{S\left(n_{0}\right)}{n_{0}^{2}}\right) .
$$

By an obvious repeated application of Lemma 2.2 one obtains the following result.

Lemma 2.3. Suppose that $n_{0} \geq 2$ and that $S$ is submultiplicative on $\left[1, n_{0}\right]$. Then, given $\varepsilon>0$ and $N_{0}>n_{0}$, there exists a submultiplicative extension of $S$ to $\left[1, N_{0}\right]$ such that $S\left(N_{0}\right)<S\left(n_{0}\right)+\varepsilon$.

Lemma 2.4. Suppose that $S$ is submultiplicative on $\left[1, n_{0}\right]$, where $n_{0} \geq 2$, and that $S\left(n_{0}\right)=$ $K \geq 2$. Then there exist $N_{0}>n_{0}$ and a submultiplicative extension of $S$ to $\left[1, N_{0}\right]$ such that $S\left(N_{0}\right) \geq 3 K / 2$.

Proof. Let $n_{1}=n_{0}^{2}$. By Lemma 2.2 we may and shall assume that $S$ has been extended to be submultiplicative on $\left[1, n_{1}\right]$. By a second application of Lemma 2.2 there exists $\varepsilon>0$ such that

$$
S_{\varepsilon}(x)= \begin{cases}S(x) & \text { for } 1 \leq x \leq n_{1} \\ S\left(n_{1}\right)+\varepsilon\left(x-n_{1}\right) & \text { for } n_{1}<x \leq n_{1}^{2}\end{cases}
$$

is submultiplicative on $\left[1,2 n_{1}\right]$ (or even $\left[1, n_{1}^{2}\right]$ although we will only use submultiplicativity on $\left.\left[1,2 n_{1}\right]\right)$. If $S_{\varepsilon}\left(2 n_{1}\right) \geq 3 K / 2$ then we are done. So we may assume that $S\left(2 n_{1}\right)<3 K / 2$, which implies (since $S\left(n_{1}\right) \geq K$ ) that

$$
n_{1} \varepsilon<\frac{K}{2}
$$

Choose $N_{0}>2 n_{1}$ such that $S_{\varepsilon}\left(N_{0}\right)=3 K / 2$. We shall show that $S_{\varepsilon}$ is submultiplicative on $\left[1, N_{0}\right]$. So suppose that $1 \leq x \leq y \leq x y \leq N_{0}$. Since $S_{\varepsilon}(x)$ is submultiplicative on $\left[1,2 n_{1}\right]$ we may assume that $x y \geq 2 n_{1}$. Since $n_{1}=n_{0}^{2}$, it follows that $y \geq n_{0}$, so $S_{\varepsilon}(y) \geq K$. First consider the case $x>2$. Then $S_{\varepsilon}(x) \geq 2$, so

$$
S_{\varepsilon}(x y) \leq S_{\varepsilon}\left(N_{0}\right)<2 K \leq S_{\varepsilon}(x) S_{\varepsilon}(y) .
$$


On the other hand, if $1 \leq x \leq 2$, then by condition (b) of Definition $2.1 S_{\varepsilon}(x)=x$, so the submultiplicativity condition becomes

$$
S_{\varepsilon}(y)+\varepsilon(x-1) y=S_{\varepsilon}(x y) \leq S_{\varepsilon}(x) S_{\varepsilon}(y)=x S_{\varepsilon}(y),
$$

i.e. $\varepsilon y \leq S_{\varepsilon}(y)$. This is clearly satisfied if $n_{0} \leq y \leq K / \varepsilon$ since $S_{\varepsilon}(y) \geq S_{\varepsilon}\left(n_{0}\right)=K$. But

$$
\begin{aligned}
S_{\varepsilon}\left(\frac{K}{\varepsilon}\right) & =S_{\varepsilon}\left(n_{1}\right)+\varepsilon\left(\frac{K}{\varepsilon}-n_{1}\right) \\
& \geq K+K-n_{1} \varepsilon \geq K+K-\frac{K}{2} \\
& =\frac{3 K}{2}=S_{\varepsilon}\left(N_{0}\right),
\end{aligned}
$$

where the last inequality follows from (2.3). Thus, $K / \varepsilon \geq N_{0}$, which proves that $S_{\varepsilon}$ is submultiplicative on $\left[1, N_{0}\right]$ as desired.

By an obvious repeated application of Lemma 2.4 one obtains the following result.

Lemma 2.5. Suppose that $n_{0} \geq 2$ and that $S$ is submultiplicative on $\left[1, n_{0}\right]$. Then, given $M>0$, there exist $N_{0}>n_{0}$ and a submultiplicative extension of $S$ to $\left[1, N_{0}\right]$ such that $S\left(N_{0}\right)>M$.

Next we construct an infinite collection of mutually incomparable submultiplicative functions. This will be used to construct spreading model diagrams in Theorems 2.9 and 2.10 below. (In fact, the existence of arbitrarily large finite sets of incomparable submultiplicative functions would suffice for the applications.)

Proposition 2.6. There exists a sequence $\left(S_{i}\right)_{i=1}^{\infty}$ of submultiplicative functions on $[1, \infty)$ such that for every nonempty finite set $A \subset \mathbb{N}$ and for every $j \in \mathbb{N} \backslash A$, we have

$$
\sup _{n \geq 1} \frac{S_{j}(n)}{\max _{i \in A} S_{i}(n)}=\infty \text {. }
$$

Proof. We shall define $\left(S_{i}\right)_{i=1}^{\infty}$ on $[1, \infty)$ by defining their values inductively on an increasing sequence of initial segments $\left[1, n_{0}\right]$. Let us describe the inductive step. Suppose that $\left(S_{i}\right)$ have been defined to be submultiplicative on some initial segment $\left[1, n_{0}\right]$ in such a way that the collection of restrictions of $\left(S_{i}\right)$ to $\left[1, n_{0}\right]$ is a finite collection of functions on $\left[1, n_{0}\right]$. Now fix a finite set $A \subseteq \mathbb{N}$ and a positive integer $N$. By applying Lemma 2.3 to $S_{i}(i \in A)$ on $\left[1, n_{0}\right]$, and applying Lemma $\left[2.5\right.$ to $S_{i}(i \in \mathbb{N} \backslash A)$ on $\left[1, n_{0}\right]$ (which is a finite collection by assumption), there exist $N_{0}>n_{0}$ and submultiplicative extensions of $S_{i}$ to $\left[1, N_{0}\right]$ such that

$$
\max _{n \in\left[n_{0}, N_{0}\right]} \frac{S_{j}(n)}{\max _{i \in A} S_{i}(n)}>N
$$


for all $j \in \mathbb{N} \backslash A$. At the end of the inductive step we have defined $\left(S_{i}\right)$ to be submultiplicative on $\left[1, N_{0}\right]$. Moreover, the new collection of initial segments of $\left(S_{i}\right)_{i=1}^{\infty}$ on $\left[1, N_{0}\right]$ thus obtained will be finite. Now one simply enumerates (in any manner) the countable collection of possible choices for $A$ and $N$ to carry out the inductive definition.

Let $1 \leq p<\infty$ and let $w=(w(n))_{n=1}^{\infty}$ be a non-increasing sequence of positive weights such that $w(1)=1, w(n) \rightarrow 0$ as $n \rightarrow \infty$, and $\sum_{n=1}^{\infty} w(n)=\infty$. Recall that the Lorentz sequence space $d(w, p)$ is the Banach space with Schauder basis $\left(e_{n}\right)$ whose norm is defined by

$$
\left\|\sum_{n=1}^{\infty} a_{n} e_{n}\right\|_{w, p}:=\left(\sum_{n=1}^{\infty} a_{n}^{* p} w(n)\right)^{1 / p},
$$

where $\left(a_{n}^{*}\right)_{n=1}^{\infty}$ is the nonincreasing rearrangement of any scalar sequence $\left(\left|a_{n}\right|\right)_{n=1}^{\infty}$ which converges to zero. Note that

$$
\left\|\sum_{n=1}^{\infty} a_{n} e_{n}\right\|_{w, p} \leq\left\|\sum_{n=1}^{\infty} a_{n} e_{n}\right\|_{p}:=\left(\sum_{n=1}^{\infty}\left|a_{n}\right|^{p}\right)^{1 / p} .
$$

The corresponding fundamental function $(S(n))_{n=1}^{\infty}$ is defined by

$$
S(n)=\left\|\sum_{i=1}^{n} e_{i}\right\|_{w, p}^{p}=\sum_{i=1}^{n} w(i) .
$$

It is known that $d(w, p)$ contains subspaces that are almost isometric to $\ell_{p}$ and is reflexive if and only if $1<p<\infty$.

The weight $w$ is said to be submultiplicative if there exists a constant $C$ such that $S(m n) \leq$ $C S(m) S(n)$ for all $m, n \in \mathbb{N}$. We require the following theorem due to Altshuler, Casazza and Lin.

Fact 2.7. ACL Suppose that $w$ is submultiplicative. Then every normalized block basis in $d(w, p)$ has a subsequence which is equivalent to the unit vector basis of $\ell_{p}$ or to the unit vector basis of $d(w, p)$.

The above theorem has the following immediate corollary.

Corollary 2.8. Suppose that $w$ is submultiplicative. Then every spreading model of $d(w, 1)$ generated by a weakly null sequence is equivalent to the unit vector basis of $d(w, 1)$. For $1<$ $p<\infty$, every spreading model of $d(w, p)$ generated by a weakly null sequence is equivalent to the unit vector basis of $\ell_{p}$ or to the unit vector basis of $d(w, p)$.

Note that to each submultiplicative function $S$ defined on $[1, \infty)$ there corresponds a submultiplicative weight sequence $w(n):=S(n)-S(n-1)$ (with constant $C=1$ ) whose 
fundamental function is $(S(n))_{n=1}^{\infty}$. Let $w_{i}(1 \leq i<\infty)$ be the weight sequences corresponding to the submultiplicative functions $S_{i}$ constructed in Proposition 2.6. Note that $\lim _{n \rightarrow \infty} w_{i}(n)=0$ for each $i$.

Now we come to the main results of this section. For $n \in \mathbb{N}$, let $P(n)$ denote the power set of $\{1, \ldots, n\}$ partially ordered by inclusion.

Theorem 2.9. For each $n \in \mathbb{N}$, let $X_{n}(1):=\left(\sum_{i=1}^{n} \oplus d\left(w_{i}, 1\right)\right)_{\infty}$. Then $S P_{w}\left(X_{n}(1)\right)$ is order-isomorphic to $P(n) \backslash\{\emptyset\}$.

Proof. Let $\left(f_{j}\right)_{j=1}^{\infty}$ be a normalized spreading model for $X_{n}(1)$ generated by a weakly null sequence. Then there exist a nonempty $A \subseteq\{1, \ldots, n\}$ and normalized spreading models $\left(f_{j}^{i}\right)_{j=1}^{\infty}$ of $d\left(w_{i}, 1\right)(i \in A)$, generated by weakly null sequences in $d\left(w_{i}, 1\right)$, such that

$$
\left\|\sum_{j=1}^{\infty} a_{j} f_{j}\right\| \approx \max _{i \in A}\left\|\sum_{j=1}^{\infty} a_{j} f_{j}^{i}\right\| .
$$

Thus, by the first part of Corollary 2.8 .

$$
\left\|\sum_{j=1}^{\infty} a_{j} f_{j}\right\| \approx \max _{i \in A}\left\|\sum_{j=1}^{\infty} a_{j} e_{j}\right\|_{w_{i}, 1} .
$$

Conversely, the right-hand side of (2.5) defines a normalized spreading model $S P(A)$ for every nonempty $A \subseteq\{1, \ldots, n\}$. Note that

$$
\left\|\sum_{j=1}^{m} f_{j}\right\| \approx \max _{i \in A} S_{i}(m) \quad(m \in \mathbb{N}) .
$$

Thus, by (2.4) of Proposition 2.6, we have

$$
A \subset B \Leftrightarrow S P(A)<S P(B)
$$

for all nonempty $A, B \subseteq\{1, \ldots, n\}$.

In the reflexive case $(1<p<\infty)$ we have to add an extra node on the top.

Theorem 2.10. Let $1<p<\infty$ and, for each $n \in \mathbb{N}$, let $X_{n}(p):=\left(\sum_{i=1}^{n} \oplus d\left(w_{i}, p\right)\right)_{\infty}$. Then $S P_{w}\left(X_{n}(p)\right)$ is order-isomorphic to $(P(n) \cup\{\{1, \ldots, n+1\}\}) \backslash\{\emptyset\}$.

Proof. The proof is essentially the same as before. However, from the second part of Corollary 2.8, we obtain an extra spreading model equivalent to the unit vector basis of $\ell_{p}$ which dominates every other spreading model. This spreading model corresponds to $\{1, \ldots, n+1\}$ under the order-isomorphism. 


\section{Countable lattices with a minimum element}

Recall that a lattice is a partially ordered set in which any two elements have both a least upper bound and a greatest lower bound. The following theorem is the main result of this section.

Theorem 3.1. Let $L$ be a countable lattice with a minimum element not containing an infinite increasing sequence. Then there exists a reflexive space $X_{L}$ such that $\operatorname{SP}_{w}\left(X_{L}\right)$ is order-isomorphic to $L$.

Remark 3.2. Recall that $\left(S P_{w}(X), \leq\right)$ is always a semi-lattice, i.e. every two elements have a least upper bound (Fact 1.1), and that when countable it does not contain any infinite increasing sequences (Fact 1.31). It is easy to see that such a semi-lattice with a minimum element is automatically a lattice. Thus, Theorem 3.1 characterizes the possible poset structure of $\left(S P_{w}(X), \leq\right)$ when $S P_{w}(X)$ is countable and has a minimum element.

The space $X_{L}$ will be an $\ell_{p}$ direct-sum of suitably constructed Orlicz sequence spaces. The proof of the theorem will be given at the end of the section. First we recall some preliminary facts about Orlicz spaces. All the unexplained terms and facts can be found in Chapter 4 of [LT], with which our notation is consistent.

An Orlicz function $M$ is a real-valued continuous non-decreasing and convex function defined on $[0,1]$ such that $M(0)=0$ and $M(1)=1$. For a given $M$, the Orlicz sequence space $\ell_{M}$ is the space of all sequences of scalars $x=\left(a_{1}, a_{2}, \ldots\right)$ such that $\sum_{n=1}^{\infty} M\left(\left|a_{n}\right| / \rho\right)<\infty$ for some $\rho>0$, equipped with the norm

$$
\|x\|=\inf \left\{\rho>0: \sum_{n=1}^{\infty} M\left(\left|a_{n}\right| / \rho\right) \leq 1\right\} .
$$

We will always assume that $M$ satisfies the $\Delta_{2}$-condition at zero (i.e., that there exists $C>0$ such that $M(2 t) \leq C M(t)$ for all $0 \leq t \leq 1 / 2)$. Then the unit vectors form a normalized symmetric basis for $\ell_{M}$. If $N$ also satisfies the $\Delta_{2}$-condition at zero then $M$ and $N$ are equivalent if there exists a constant $C>0$ such that $(1 / C) N(t) \leq M(t) \leq C N(t)$ for all $0 \leq t \leq 1$

If $C \geq 1$ and $M$ and $N$ are two Orlicz functions such that $N(t) \leq C M(t)$ for all $0<t \leq 1$, then the unit vector basis of $\ell_{M} C$-dominates that of $\ell_{N}$. Conversely, if $M$ and $N$ satisfy the $\Delta_{2}$-condition at zero and the unit vector basis of $\ell_{M}$ dominates that of $\ell_{N}$ then there exists $C \geq 1$ such that $N(t) \leq C M(t)$ for all $0<t \leq 1$.

If $M$ satisfies the $\Delta_{2}$-condition at zero then an Orlicz sequence space $\ell_{N}$ is isomorphic to a subspace of $\ell_{M}$ if and only if $N$ is equivalent to some function in $C_{M, 1}$, where $C_{M, 1}$ is 
the norm-closed convex hull in $C[0,1])$ of the set

$$
E_{M, 1}=\overline{\left\{\frac{M(\lambda t)}{M(\lambda)} ; 0<\lambda<1\right\}}
$$

See [LT, Lemma 4.a.6 and remark (p. 141)] for this result.

As noted in [S1, this is easily generalized to the spreading models of $\ell_{M}:\left(\tilde{x}_{i}\right)$ is a spreading model generated by a normalized block sequence $\left(x_{i}\right)$ in $\ell_{M}$ if and only if $\left(\tilde{x}_{i}\right)$ is isometrically equivalent to the unit vector basis of $\ell_{N}$ for some $N \in C_{M, 1}$.

We will use the following method of representing Orlicz functions by sequences of zeros and ones, introduced by Lindenstrauss and Tzafriri [LT, p. 161].

Fix $0<\tau<1$ and $1<r<p<\infty$. For every sequence of zeros and ones, $\eta=(\eta(n))_{n=1}^{\infty}$ (i.e. $\eta(n) \in\{0,1\}$ for all $n$ ), let $M_{\eta}$ be the piecewise linear function defined on $[0,1]$ satisfying $M_{\eta}(0)=0, M_{\eta}(1)=1$, and

$$
M_{\eta}\left(\tau^{k}\right)=\tau^{r k+(p-r) \sum_{n=1}^{k} \eta(n)}, \quad k=1,2, \ldots
$$

Lemma 3.3. Fix $0<\tau<1$ and $1<r<p<\infty$. If $p-r$ is sufficiently small, then, for all $\eta, M_{\eta}$ is an Orlicz function satisfying the $\Delta_{2}$-condition at zero.

Proof. To show that $M_{\eta}$ is convex it suffices to check that the slope of the chord joining $\left(\tau^{n+1}, M_{\eta}\left(\tau^{n+1}\right)\right)$ to $\left(\tau^{n}, M_{\eta}\left(\tau^{n}\right)\right)$ is a decreasing function of $n$, i.e.

$$
\frac{M_{\eta}\left(\tau^{n}\right)-M_{\eta}\left(\tau^{n+1}\right)}{\tau^{n}-\tau^{n+1}} \leq \frac{M_{\eta}\left(\tau^{n-1}\right)-M_{\eta}\left(\tau^{n}\right)}{\tau^{n-1}-\tau^{n}}
$$

Using the fact that $M_{\eta}\left(\tau^{k+1}\right)=\tau^{r} M_{\eta}\left(\tau^{k}\right)$ if $\eta(k+1)=0$ and $M_{\eta}\left(\tau^{k+1}\right)=\tau^{p} M_{\eta}\left(\tau^{k}\right)$ if $\eta(k+1)=1$, (3.2) simplifies to the following pair of conditions:

$$
\tau^{r-1}\left(1-\tau^{p}\right) \leq 1-\tau^{r} \quad \text { and } \quad \tau^{p-1}\left(1-\tau^{r}\right) \leq 1-\tau^{p} .
$$

Both conditions are clearly satisfied if $p-r$ is sufficiently small. (Note that the first condition is not satisfied, however, if $r$ is very close to 1 .) The $\Delta_{2}$-condition is easily checked.

Henceforth, we shall always assume that $\tau, p$, and $r$ satisfy the conclusion of Lemma 3.3 .

Proposition 3.4. Suppose that $1<p<2$. Then there exists $C<\infty$ such that for each sequence $\eta$ of zeros and ones, $\ell_{M_{\eta}} C$-embeds into $L_{1}[0,1]$.

Proof. Observe that the inequalities in (3.3) are reversed if $0<r<p<1$. This implies that $M_{\eta}(\sqrt{t})$ is equivalent to a concave function if $p<2$. By a result of Bretagnolle and Dacuhna-Castelle $[\mathrm{BD}] \ell_{M_{\eta}}$ embeds isomorphically into $L_{1}[0,1]$. To see that there is a uniform embedding constant, observe that there exists a 'universal' sequence $\rho=$ $(\rho(n))_{n=1}^{\infty}$ such that every sequence of zeros and ones, $\eta$, is a pointwise limit of the collection 
$\left\{\left(\rho(n+k)_{n=1}^{\infty}: k \in \mathbb{N}\right\}\right.$ of left shifts of $\rho$ : indeed, let $\rho$ be the concatenation of all possible finite sequences of zeros and ones. It follows that $E_{M_{\rho}, 1}$ (see (3.1) ) contains $M_{\eta}$ for every $\eta$, and hence that $\ell_{M_{\eta}}$ is isometric to a spreading model of $\ell_{M_{\rho}}$ for every $\eta$. Finally, since $\ell_{M_{\rho}} C$-embeds into $L_{1}[0,1]$ for some $C<\infty$, it follows that $\ell_{M_{\eta}} C$-embeds into $L_{1}[0,1]$ for every $\eta$.

We will be interested in only a simple class of such spaces as described in the following.

Lemma 3.5. Let $1<r<p<\infty$, and $0<\tau<1$. Let $\left(n_{k}\right) \subset \mathbb{N}$ satisfy $n_{1}=1$ and $n_{k+1}-n_{k} \uparrow \infty$, and put

$$
\rho(i)= \begin{cases}0 & \text { if } i=n_{k} \\ 1 & \text { otherwise }\end{cases}
$$

Let $M:=M_{\rho}$ be the corresponding Orlicz function. Then $\ell_{M}$ satisfies the following:

(a) Every spreading model is $\tau^{-2 p}$-dominated by the unit vector basis of $\ell_{M}$.

(b) Every spreading model is equivalent either to the unit vector basis of $\ell_{M}$ or to the unit vector basis of $\ell_{p}$.

(c) Every spreading model that is equivalent to the unit vector basis of $\ell_{p}$ is actually $\tau^{-5 p}$-equivalent to the unit vector basis of $\ell_{p}$.

(d) $\ell_{M}$ is reflexive.

Proof. Observe that

$$
\sum_{i=1}^{n} \rho(i) \leq \sum_{i=k+1}^{k+n} \rho(i) \text { for all } k, n \in \mathbb{N} .
$$

Therefore, for all $\lambda=\tau^{k}$ and $t=\tau^{n}$, we have

$$
\begin{aligned}
\frac{M(\lambda t)}{M(\lambda)} & =\frac{\tau^{r(k+n)+(p-r) \sum_{i=1}^{k+n} \rho(i)}}{\tau^{r k+(p-r) \sum_{i=1}^{k} \rho(i)}} \\
& =\tau^{r n+(p-r) \sum_{i=k+1}^{k+n} \rho(i)} \leq \tau^{r n+(p-r) \sum_{i=1}^{n} \rho(i)}=M(t) .
\end{aligned}
$$

A simple calculation now yields, for all $0<\lambda, t<1$, that

$$
\frac{M(\lambda t)}{M(\lambda)} \leq \tau^{-2 p} M(t)
$$

Now let $N \in C_{M, 1}$. Then, by the definition of $C_{M, 1}, N$ is the limit in the uniform norm of a sequence of convex combinations $\left(F_{n}\right)$ of the form

$$
F_{n}=\sum_{i \in A_{n}} a_{i} \frac{M\left(\lambda_{i} t\right)}{M\left(\lambda_{i}\right)},
$$

for some finite $A_{n} \subset \mathbb{N}, 0<\lambda_{i} \leq 1$, and positive $\left(a_{i}\right)$ with $\sum_{i \in A_{n}} a_{i}=1$. 
Thus (3.4) implies that for all $N \in C_{M, 1}$,

$$
N(t) \leq \tau^{-2 p} M(t) \text { for all } 0<t<1,
$$

which proves (a). To see that $N$ is equivalent either to $M$ or to $t^{p}$, we distinguish two cases corresponding to the manner in which the sequence $\left(F_{n}\right)$ converges to $N$.

For the first case, suppose that there exists $n_{0} \in \mathbb{N}, \bar{\lambda}>0$ and $\delta>0$ such that for all $n \geq n_{0}$,

It follows that

$$
\sum_{\lambda_{i} \geq \bar{\lambda}, i \in A_{n}} a_{i} \geq \delta
$$

$$
N(t) \geq \delta M(\bar{\lambda} t), \text { for all } t>0,
$$

which, along with (3.5), implies that $N$ is equivalent to $M$.

For the second case, we suppose that for all $n_{0} \in \mathbb{N}, \bar{\lambda}>0$ and $\delta>0$, there exists $n \geq n_{0}$ such that

$$
\sum_{\lambda_{i} \geq \bar{\lambda}, i \in A_{n}} a_{i}<\delta
$$

Fix $t=\tau^{m}$. Since $n_{k+1}-n_{k} \uparrow \infty$, it follows that every $m$ consecutive terms of $\rho$ which begin sufficiently far along the sequence can contain at most one zero term. This implies that if $\lambda=\tau^{k}$ is sufficiently small then

$$
t^{p} \leq \frac{M(\lambda t)}{M(\lambda)} \leq \tau^{-p} t^{p}
$$

Now fix $\delta>0$ and $0<t<1$. It follows easily from (3.6) that there exists $\bar{\lambda}>0$ such that for all $\lambda<\bar{\lambda}$

$$
\tau^{2 p} t^{p} \leq \frac{M(\lambda t)}{M(\lambda)} \leq \tau^{-3 p} t^{p}
$$

By assumption there exists $n \in \mathbb{N}$ such that

$$
\left|F_{n}(t)-N(t)\right|<\delta \text { and } \sum_{\lambda_{i} \geq \bar{\lambda}, i \in A_{n}} a_{i}<\delta .
$$

Now (3.7) gives

$$
F_{n}(t)=\sum_{\lambda_{i}<\bar{\lambda}, i \in A_{n}} a_{i} \frac{M\left(\lambda_{i} t\right)}{M\left(\lambda_{i}\right)}+\sum_{\lambda_{i} \geq \bar{\lambda}, i \in A_{n}} a_{i} \frac{M\left(\lambda_{i} t\right)}{M\left(\lambda_{i}\right)} \leq \tau^{-3 p} t^{p}+\delta .
$$

A similar calculation yields

$$
F_{n}(t) \geq(1-\delta) \tau^{2 p} t^{p}-\delta
$$

Since $\left|N(t)-F_{n}(t)\right|<\delta$ and $\delta>0$ is arbitrary, we get

$$
\tau^{2 p} t^{p} \leq N(t) \leq \tau^{-3 p} t^{p} .
$$


This proves (c) and also completes the proof of (b). Finally, (b) and [LT, Lemma 4.a.6] imply that $\ell_{1}$ is not isomorphic to a subspace of $\ell_{M}$, which in turn implies by $\mathrm{LT}$, Proposition 4.a.4] that $\ell_{M}$ is reflexive, which proves (d).

We will also make use of the following general fact.

Lemma 3.6. Let $X=\left(\sum_{j=1}^{\infty} \oplus X_{j}\right)_{p}$, where $1 \leq p<\infty$ and each $X_{j}$ is an infinitedimensional Banach space, and let $\left(\tilde{x}_{i}\right) \in S P_{w}(X)$ be a spreading model generated by a normalized weakly null sequence in $X$. Then there exist non-negative $\left(c_{j}\right)_{j=0}^{\infty}$ with $\sum_{j=0}^{\infty} c_{j}^{p}=$ 1 and normalized spreading models $\left(\tilde{x}_{i}^{j}\right)_{i} \in S P_{w}\left(X_{j}\right)$ such that for all scalars $\left(a_{i}\right)$

$$
\left\|\sum_{i} a_{i} \tilde{x}_{i}\right\|=\left[\sum_{j=1}^{\infty} c_{j}^{p}\left\|\sum_{i} a_{i} \tilde{x}_{i}^{j}\right\|^{p}+c_{0}^{p} \sum_{i}\left|a_{i}\right|^{p}\right]^{1 / p} .
$$

Proof. Suppose that the normalized weakly null sequence $\left(y_{i}\right)$ generates the spreading model $\left(\tilde{x}_{i}\right)$. Write $y_{i}=\left(y_{i}^{j}\right)_{j=1}^{\infty}$, where $y_{i}^{j} \in X_{j}$ for each $j$. By a diagonalization argument, $\left(y_{i}\right)$ has a subsequence $\left(x_{i}\right)$ such that

$$
\lim _{i \rightarrow \infty}\left\|x_{i}^{j}\right\|=c_{j} \text { and } \sup _{i \geq j}\left|\left\|x_{i}^{j}\right\|-c_{j}\right| \leq \frac{1}{2^{j}}
$$

and $\left(c_{j}^{-1} x_{i}^{j}\right)_{i=1}^{\infty}$ generates a normalized spreading model $\left(\tilde{x}_{i}^{j}\right)_{i} \in S P_{w}\left(X_{j}\right)$. Note that (3.9) implies that $c_{0}:=\lim _{i \rightarrow \infty}\left\|x_{i}-P_{i}\left(x_{i}\right)\right\|$ exists, where $P_{i}\left(x_{i}\right)=\left(x_{i}^{1}, x_{i}^{2}, \ldots, x_{i}^{i}, 0,0,0, \ldots\right)$. One now checks that the spreading model $\left(\tilde{x}_{i}\right)$ generated by $\left(x_{i}\right)$ is given by the stated formula. (Note also that $\sum_{j=0}^{\infty} c_{j}^{p}=1$ since $\left(x_{i}\right)$ is normalized.)

Before proving Theorem 3.1 we give an application of Lemma 3.6

Theorem 3.7. Let $X$ be an infinite-dimensional Banach space such that $S P_{w}(X)$ is a countable chain. Then there exists a countable ordinal $\alpha$ such that $S P_{w}(X)$ is order-isomorphic to $\alpha$ with the reverse order. Conversely, if $\alpha \geq 1$ is a countable ordinal then there exists a reflexive Banach space $X$ such that $S P_{w}(X)$ is order-isomorphic to $\alpha$ with the reverse order.

Proof. For the first part, by Fact $1.3 S P_{w}(X)$ does not admit an infinite strictly increasing sequence. Thus the reverse order on $S P_{w}(X)$ is a well-ordering and hence is orderisomorphic to a countable ordinal. For the converse, let $\beta \mapsto p_{\beta}(\beta<\alpha)$ be an increasing order-isomorphism from $\alpha$ onto a subset of $[2,3]$ such that $p_{0}=2$, and set $X:=\left(\sum_{\beta<\alpha} \oplus \ell_{p_{\beta}}\right)_{2}$. Using the well-foundedness of $\alpha$ and the monotonicity property of the $\ell_{p}$ norms (i.e., that $\|\cdot\|_{q} \leq\|\cdot\|_{p}$ if $p \leq q$ ), it follows easily from Lemma 3.6 that 
every normalized spreading model of $X$ is equivalent to the unit vector basis of $\ell_{p_{\beta}}$ for some $\beta<\alpha$; so $S P_{w}(X)$ is order-isomorphic to $\alpha$ with the reverse order.

We now proceed to the

Proof of Theorem 3.1. For convenience we shall assume that $L$ is countably infinite. (When $L$ is finite only minor notational changes are needed.) The space $X_{L}$ will be of the form $X_{L}=\left(\sum_{j=0}^{\infty} \oplus \ell_{M_{j}}\right)_{p}$ for suitably constructed Orlicz sequence space $\ell_{M_{j}}$ 's, with $M_{j}:=M_{\rho_{j}}$ for certain sequences $\rho_{j}$ of zeros and ones (for the same $\tau, r$, and $p$ ). The 'patterns' of the $\rho_{j}$ 's will be of the form

$$
\rho_{j}(i)= \begin{cases}0 & \text { if } i \in \sigma(j) \\ 1 & \text { otherwise. }\end{cases}
$$

for some fast increasing sequence $\sigma(j) \subset \mathbb{N}$, with $1 \in \sigma(j)$. For simplicity, for every $j$ we will take $\sigma(j)$ to be a subset of $\mathcal{M}=\left\{1,2,2^{2}, 2^{3}, \ldots\right\}$ which will ensure that the hypothesis of Lemma 3.5 is satisfied.

The patterns of the $\rho_{j}$ 's (equivalently, the $\sigma_{j}$ 's) will be developed inductively on finite intervals of $\mathbb{N}$ according to a two-step procedure which we call $(\varepsilon, A)$-domination.

Let $A \subset \mathbb{N}$ and $\varepsilon>0$. Suppose that for some $N \in \mathbb{N}$, the $\rho_{j}$ 's have already been defined on the initial segment $[1, N]$ so that

$$
\sum_{i=1}^{N} \rho_{j}(i)=\sum_{i=1}^{N} \rho_{k}(i), \text { for all } j, k \in \mathbb{N} .
$$

The $(\varepsilon, A)$-domination procedure extends the definition of the $\rho_{j}$ 's to an intial segment $\left[1, N_{1}\right]$ for some $N_{1}>N$. Let us first dispose of some trivial cases. If $A=\emptyset$ or if $A=\mathbb{N}$ then set $N_{1}=N+1$ and $\rho_{j}\left(N_{1}\right)=1$ for all $j$.

Now suppose that both $A$ and $\mathbb{N} \backslash A$ are non-empty. The first step of the procedure is carried out as follows. Choose a sufficiently large (just how large is specified below) integer $m>N$. For all $k \in \mathbb{N} \backslash A$ place 0's on the coordinates from $[N+1, m] \cap \mathcal{M}$ of the $\rho_{k}$ 's (while the rest of the coordinates of the interval are filled with 1's), and for all $j \in A$ place 1 's on all the coordinates from $[N, m]$ of the $\rho_{j}$ 's , where $m$ is chosen so that

$$
\sum_{i=1}^{m} \rho_{j}(i)-\sum_{i=1}^{m} \rho_{k}(i)
$$

is sufficiently large to ensure that

$$
\frac{M_{\rho_{j}}\left(\tau^{m}\right)}{M_{\rho_{k}}\left(\tau^{m}\right)}<\varepsilon, \text { for all } j \in A, k \in \mathbb{N} \backslash A \text {. }
$$

For the second step we choose a sufficiently large integer $N_{1}>m$ (just how large is specified below), with $N_{1} \in \mathcal{M}$, and 'rebalance' all of the $\rho_{j}$ 's on the interval $\left[m+1, N_{1}\right]$. This is 
achieved by placing 0's on the coordinates from $\left[m+1, N_{1}\right] \cap \mathcal{M}$ for all the $\rho_{j}$ 's $(j \in A)$ and by placing 1's on the coordinates from $\left[m+1, N_{1}\right]$ for all the $\rho_{k}$ 's $(k \in \mathbb{N} \backslash A)$, where $N_{1}$ is chosen so that (3.10) is satisfied with $N$ replaced by $N_{1}$. At the end of this second step the $M_{j}$ 's are equal again, i.e.

$$
M_{j}\left(\tau^{N_{1}}\right)=M_{k}\left(\tau^{N_{1}}\right) \quad \text { for all } j . k \in \mathbb{N}
$$

We now pass to the main construction. Let $L=\left\{e_{0}, e_{1}, e_{2}, \ldots,\right\}$ be the given countable lattice, where $e_{0}$ is the minimum element. Consider $\bar{L}=\left\{\bar{e}_{1}, \bar{e}_{2}, \ldots\right\}$, where $\bar{e}_{j}=\{i \in \mathbb{N}$ : $\left.e_{i} \leq e_{j}\right\}$ for all $j \in \mathbb{N}$. Put $\rho_{0}=(1,1,1, \ldots)$.

We begin by setting $\rho_{j}(1)=0$ for all $j \in \mathbb{N}$, which ensures that the $\rho_{j}^{\prime} s$ satisfy Lemma 3.5. Now, for every $j \in \mathbb{N}$ and every $\varepsilon=2^{-k}, k=1,2, \ldots$, we carry out an $(\varepsilon, A)$-domination procedure for $A=\bar{e}_{j}$. Since there are countably many choices we can enumerate some order in which to carry out all $(\varepsilon, A)$-dominations.

The resulting sequences $\rho_{0}, \rho_{1}, \rho_{2}, \ldots$ have the following properties.

(i) $M_{\rho_{0}}$ is equivalent to the function $t^{p}$.

(ii) For all $i, j \in \mathbb{N} \cup\{0\}$, there exists a constant $C<\infty$ such that

$$
M_{\rho_{i}}(t) \leq C M_{\rho_{j}}(t) \text { for all } 0<t<1
$$

if and only if $e_{i} \leq e_{j}$ in $(L, \leq)$. Moreover, if there exists such a $C$ then $C=1$ works.

(iii) For every non-empty finite set $F \subset \mathbb{N} \cup\{0\}$

$$
\max _{j \in F} M_{\rho_{j}}=M_{\rho_{j_{0}}}, \text { where } e_{j_{0}}=\bigvee_{j \in F} e_{j} .
$$

Proof of (iii). To derive a contradiction, assume that there exists $t=\tau^{m}$ such that $\max _{j \in F} M_{\rho_{j}}(t)<M_{\rho_{j_{0}}}(t)$. Because of the 'rebalancing' step in the domination procedure, it follows that $m$ belongs to an interval of $\mathbb{N}$ where an $(\varepsilon, A)$-domination takes place for some $A$ such that $F \subseteq A$ and $j_{0} \in \mathbb{N} \backslash A$. There exists $k \in \mathbb{N}$ such that $A=\bar{e}_{k}$. Then $e_{j} \leq e_{k}$ for all $j \in F$. Since $L$ is a lattice it follows that $e_{j_{0}} \leq e_{k}$, and hence $j_{0} \in A$, which is the desired contradiction.

(iv) Let $B$ be a non-empty subset of $\mathbb{N} \cup\{0\}$ Then there exists a finite subset $F$ of $B$ such that

$$
\max _{j \in B} M_{\rho_{j}}=\max _{j \in F} M_{\rho_{j}} .
$$

Proof of (iv). Suppose not. Then there exists $\left(j_{k}\right)_{k=1}^{\infty} \subset B$ such that for all $n \in \mathbb{N}$

$$
\max _{1 \leq k \leq n} M_{\rho_{j_{k}}}<\max _{1 \leq k \leq n+1} M_{\rho_{j_{k}}}
$$


This, however, implies by (iii) that

$$
\bigvee_{1 \leq k \leq n} e_{j_{k}}<\bigvee_{1 \leq j \leq n+1} e_{j_{k}}, \text { for each } n
$$

But this contradicts our assumption that there are no increasing infinite sequences in $L$.

Now consider

$$
X_{L}=\left(\sum_{j=0}^{\infty} \oplus \ell_{M_{j}}\right)_{p}
$$

where $M_{j}=M_{\rho_{j}}, j \in \mathbb{N} \cup\{0\}$. By (d) of Lemma 3.5 each $\ell_{M_{j}}$ is reflexive and hence $X_{L}$ is also reflexive. It follows from property (ii) that the collection of spreading models generated by the unit vector bases of each $\ell_{M_{j}}$ is order-isomorphic to $L$. Therefore it remains to show that every spreading model of $X_{L}$ is equivalent to the unit vector basis $\left(b_{i}^{j}\right)_{i}$ of $\ell_{M_{j}}$ for some $j \in \mathbb{N} \cup\{0\}$.

Let $\left(\tilde{x}^{i}\right)$ be a normalized spreading model of $X_{L}$. Then, for all $\left(a_{i}\right) \in c_{00}$, we have by Lemma 3.6

$$
\left\|\sum_{i} a_{i} \tilde{x}_{i}\right\|=\left[\sum_{j} c_{j}^{p}\left\|\sum_{i} a_{i} \tilde{x}_{i}^{j}\right\|^{p}+c_{0}^{p} \sum_{i}\left|a_{i}\right|^{p}\right]^{1 / p}
$$

where $\left(\tilde{x}_{i}^{j}\right)_{i}$ is a normalized spreading model of $\ell_{M_{j}}$ and $\left(c_{j}\right)_{j=0}^{\infty}$ belongs to the non-negative unit sphere of $\ell_{p}$.

Let $B$ be the collection of all $j \in \mathbb{N}$ such that $c_{j} \neq 0$ and such that $\left(\tilde{x}_{i}^{j}\right)_{i}$ is equivalent to $\left(b_{i}^{j}\right)_{i}$. If $j \notin B$ then either $c_{j}=0$ or, by Lemma 3.5. $\left(\tilde{x}_{i}^{j}\right)_{i}$ is $\tau^{-5 p}$-equivalent to the unit vector basis of $\ell_{p}$. Thus, if $B=\emptyset$, then (3.11) implies that $\left(\tilde{x}^{i}\right)$ is equivalent to the unit vector basis of $\ell_{p}$ and hence to $\left(b_{i}^{0}\right)_{i}$. So suppose that $B \neq \emptyset$. Then, by Lemma 3.5. each $\left(\tilde{x}_{i}^{j}\right)_{i}(j \in B)$ is $\tau^{-2 p}$-dominated by $\left(b_{i}^{j}\right)_{i}$. By properties (iii) and (iv) above there exist a finite set $F \subset B$ and $j_{0} \in \mathbb{N}$ such that

$$
\max _{j \in B} M_{\rho_{j}}=\max _{j \in F} M_{\rho_{j}}=M_{\rho_{j_{0}}} .
$$

Hence there exists $0 \leq K<\infty$ such that

$$
\begin{aligned}
\left\|\sum_{i} a_{i} \tilde{x}_{i}\right\| & \leq\left[\tau^{-2 p} \sum_{j \in B} c_{j}^{p}\left\|\sum_{i} a_{i} b_{i}^{j}\right\|^{p}+K \sum_{i}\left|a_{i}\right|^{p}\right]^{1 / p} \\
& \leq\left[\left(\tau^{-2 p} \sum_{j \in B} c_{j}^{p}\right)\left\|\sum_{i} a_{i} b_{i}^{j_{0}}\right\|^{p}+K \sum_{i}\left|a_{i}\right|^{p}\right]^{1 / p}
\end{aligned}
$$


which implies that $\left(\tilde{x}_{i}\right)_{i} \leq\left(b_{i}^{j_{0}}\right)$. On the other hand, there exists $c>0$ such that

$$
\begin{aligned}
\left\|\sum_{i} a_{i} \tilde{x}_{i}\right\| & \geq\left[\sum_{j \in F} c_{j}^{p}\left\|\sum_{i} a_{i} \tilde{x}_{i}^{j}\right\|^{p}\right]^{1 / p} \\
& \geq c \max _{j \in F}\left\|\sum_{i} a_{i} b_{i}^{j}\right\|
\end{aligned}
$$

(since $\left(\tilde{x}_{i}^{j}\right)_{i}$ is equivalent to $\left(b_{i}^{j}\right)_{i}$ for each $\left.j \in F\right)$

$$
\geq \frac{c}{\operatorname{card} F}\left\|\sum_{i} a_{i} b_{i}^{j_{0}}\right\|
$$

Thus, $\left(\tilde{x}_{i}\right)_{i}$ is equivalent to $\left(b_{i}^{j_{0}}\right)_{i}$.

Remark 3.8. For each $1<p<\infty$, the above construction yields the unit vector basis of $\ell_{p}$ as the minimum element of $S P_{w}\left(X_{L}\right)$. If we allow $X_{L}$ to be nonreflexive we can obtain $c_{0}$ as the minimum element. However, this requires a rather different construction. Using results of Casazza and Lin [CL, it is possible to construct a $c_{0}$-sum of duals of certain Lorentz sequence spaces for which $c_{0}$ is the minimum element of $S P_{w}\left(X_{L}\right)$. We omit the details of this result.

Remark 3.9. Let $\rho$ be the universal sequence used in the proof of Proposition 3.4 It follows from the proof of Theorem 3.1 that $S P_{w}\left(\ell_{M_{\rho}}\right)$ contains a subset that is order-isomorphic to any given countable poset $P$. (Note also that there is a universal countable poset.) By Proposition 3.4, $\ell_{M_{\rho}}$ is isomorphic to a reflexive subspace of $L_{1}[0,1]$ when $p<2$.

Corollary 3.10. For every finite lattice $L$ there exists a reflexive space $X_{L}$ such that $S P_{w}\left(X_{L}\right)$ is order-isomorphic to $L$.

Corollary 3.11. Let $L$ be a finite lattice (resp. countable lattice with a minimum element and without any infinite increasing sequence). There exists a reflexive (resp. non-reflexive) subspace $Y_{L}$ of $L_{1}[0,1]$ such that $S P_{w}\left(Y_{L}\right)$ is order-isomorphic to $L$.

Proof. Using the notation of Theorem 3.1 let

$$
Y_{L}=\left(\sum_{j=0}^{\infty} \oplus \ell_{M_{j}}\right)_{1} .
$$

By Proposition [3.4] if $p<2$ then for some $C<\infty$ each $\ell_{M_{j}} C$-embeds into $L_{1}[0,1]$, and hence $Y_{L}$ is isomorphic to a subspace of $L_{1}[0,1]$. Moreover, $Y_{L}$ is reflexive if and only if $L$ is finite. The proof of Theorem 3.1 shows that $S P_{w}\left(Y_{L}\right)$ is order-isomorphic to $L$. (Note that if $L$ is infinite then $Y_{L}$ also has an $\ell_{1}$ spreading model that is not generated by a weakly null sequence.) 


\section{REFERENCES}

[ACL] Zvi Altshuler, P. G. Casazza and Bor-Luh Lin, On symmetric basic sequences in Lorentz sequence spaces, Israel J. Math. 15 (1973), 144-155.

[AD] S. A. Argyros and I. Deliyanni, Examples of asymptotic $\ell_{1}$ Banach spaces Trans. Amer. Math. Soc. 349 (1997), no.3, 973-995.

[AOST] G. Androulakis, E. Odell, Th. Schlumprecht and N. Tomczak-Jaegermann, On the structure of the spreading models of a Banach space, Canadian J. Math., to appear.

[BL] B. Beauzamy and J.-T. Lapresté, Modèles étalés des espaces de Banach, Travaux en Cours, Herman, Paris, 1984.

[CL] P. G. Casazza and Bor-Luh Lin, On symmetric basic sequences in Lorentz sequence spaces, II, Israel J. Math. 17 (1974) 191-218.

[BD] J. Bretagnolle and D. Dacuhna-Castelle, Applications de l'étude de certaines formes linéaires aléatoires au plongement d'espaces de Banach dans les espaces $L^{p}$, Ann. Sci. École Norm. Sup. 2 (1969), 437-480.

[ER] P. Erdös and R. Rado, Combinatorial theorems on classifications of subsets of a given set, Proc. London Math. Soc. (3) 2 (1952), 417-439.

[K] J.L. Krivine, Sous espaces de dimension finie des espaces de Banach réticulés, Ann. of Math. 104 (2) (1976), 1-29.

[LT] J. Lindenstrauss and L. Tzafriri, Classical Banach Spaces vol. I: Sequence spaces, Springer-Verlag, New York, 1977.

[O1] E. Odell, On Schreier unconditional sequences, Contemporary Math. 144 (1993), 197-201.

[O2] E. Odell, Applications of Ramsey theorems to Banach space theory, Notes in Banach spaces, (H. E. lacey, ed.), Univ. Texas Press, Austin, TX, (1980) pp. 379-404.

[OS1] E. Odell and Th. Schlumprecht, A Problem on Spreading Models, J. Funct. Anal. 153 (1998), 249-261.

[OS2] E. Odell and Th. Schlumprecht, On the richness of the set of p's in Krivine's theorem, Oper. Theory: Adv. Appl. 77 (1995), 177-198.

[R] H. Rosenthal, A characterization of Banach spaces containing $\ell_{1}$, Proc. Nat. Acad. Sci. U.S.A. 71 (1974), 2411-2413.

[S1] B. Sari, On the structure of symmetric sequences in Orlicz sequence spaces, submitted.

[S2] B. Sari, On Banach spaces with few spreading models, Proc. Amer. Math. Soc., to appear.

[Sh] S. Shelah, On uncountable Boolean algebras with no uncountable pairwise comparable or incomparable sets of elements, Notre Dame J. Formal Logic 22 (1981), no.4, 301-308.

Department of Mathematics, University of South Carolina, Columbia, SC 29208-0001

E-mail address: dilworth@math.sc.edu

Department of Mathematics, The University of Texas at Austin, 1 University Station C1200, Austin, TX 78712-0257

E-mail address: odell@math.utexas.edu

Department of Mathematics, University of North Texas, Denton, TX 76203-1430

E-mail address: bunyamin@unt.edu 